\title{
Additive antitumour response to the rabbit VX2 hepatoma by combined radio frequency ablation and toll like receptor 9 stimulation
}

\author{
Barbara Behm, ${ }^{1}$ Pietro Di Fazio, ${ }^{2}$ Patrick Michl, ${ }^{3}$ Daniel Neureiter, ${ }^{4}$ Ralf Kemmerling, ${ }^{4}$ \\ Eckhart Georg Hahn, ${ }^{1}$ Deike Strobel, ${ }^{1}$ Thomas Gress, ${ }^{3}$ Detlef Schuppan, ${ }^{5,6}$ \\ Thaddaeus Till Wissniowski ${ }^{1,3}$
}

\begin{abstract}
- Additional material is published online only. To view please visit the journal online (http://dx.doi.org/10.1136/ gutjnl-2014-308286).
\end{abstract}

For numbered affiliations see end of article.

\section{Correspondence to} Dr Thaddaeus Till Wissniowski, Division of Gastroenterology, University Hospital Marburg, Baldingerstraße, Marburg 35043, Germany;

Wissniowski@icloud.com

Received 31 August 2014 Revised 10 November 2014 Accepted 11 November 2014 Published Online First 18 December 2014
CrossMark

To cite: Behm $B$, Di Fazio P, Michl $P$, et al. Gut 2016;65:134-143.

\section{ABSTRACT}

Introduction Radiofrequency ablation (RFA),

a palliative therapeutic option for solid hepatic tumours, stimulates localised and systemic antitumour cytotoxic

$\mathrm{T}$ cells. We studied how far addition of $\mathrm{CpG} B$ oligonucleotides, toll like receptor (TLR) 9 agonists, would increase the antitumoural T cell response of RFA in the highly aggressive VX2 hepatoma.

Methods Rabbits were randomised to receive RFA, $\mathrm{CpG} B$, their combination or no therapy. The antitumour efficacy of RFA alone or in combination with $C p G$ B was further tested by rechallenging a separate group with intravenously injected VX2 tumour cells after 120 days. Animals were assessed for survival, tumour size and spread, and tumour and immune related histological markers after 120 days. Peripheral blood mononuclear cells were tested for tumour-specific T cell activation and cytotoxicity. Immune modulatory cytokines tumour necrosis factor $\alpha$, interleukin (IL)-2/IL-8/IL-10/IL-12 and interferon $\gamma$, and vascular endothelial growth factor were measured in serum.

Results Mean survival of untreated animals was 36 days, as compared with 97,78 and 114 days for RFA, CpG and combination therapy, respectively. Compared with untreated controls, antitumour T cell stimulation/cytotoxicity increased 26/16-fold, 32/17-fold and 50/38-fold 2 weeks after RFA, CpG and combination treatments, respectively. The combination inhibited tumour spread to lungs and peritoneum significantly and prohibited new tumour growth in animals receiving a secondary systemic tumour cell injection. RFA alone induced a Th1 cytokine pattern, while IL-8 and IL-10 were only upregulated in CpG treated animals and controls.

Conclusions The combination of TLR9 stimulation with RFA resulted in a potentiated antitumour T cell response and cytotoxicity in the VX2 tumour model. Only this combination prevented subsequent tumour spread and resulted in a significantly improved survival, justifying the need for further exploration of the combination of ablative therapies and TLR9 agonists in liver cancer.

\section{OBJECTIVE}

Primary hepatocellular carcinoma (HCC) is the fifth most frequent cancer worldwide with a rising incidence. ${ }^{1}$ Once diagnosed, HCC carries a high short-term mortality, and only a minority of

\section{Significance of this study}

What is already known on this subject?

- Radiofrequency ablation (RFA) has become standard of care for unresectable solid hepatic tumours.

- RFA is known to have tumour-specific immunological side effects.

- ODN CpGs are already used as adjuvants to vaccines as specific toll like receptor (TLR) agonists.

- Anticancer strategies with CpG application are lacking clinical significance by missing antigens for vaccination.

What are the new findings?

- Immunological environment after CpG application alone creates a Th2/M2 immune response.

- CpGs in combination with a specific antigen source after RFA tumour ablation lead to Th1 directed immune response.

- The tumour-specific Th1 directed immune response prevents recurrence of malignant tumour growth.

How might it impact on clinical practice in the foreseeable future?

- In vivo tumour vaccination with specific TLR9 stimulation together with local tumour ablation could be an important part of future multimodal antitumour strategies.

patients can be rescued by resection or liver transplantation, leaving palliative treatment such as radiofrequency ablation (RFA), ethanol injection, transarterial chemoembolisation, chemotherapy or best supportive care for the majority of HCC patients. $^{2-5}$ Notably, most HCCs are resistant to chemotherapy, and the multikinase inhibitor sorafenib, while being approved for treatment of HCC, prolongs life by only a few months and with significant side effects. ${ }^{6}$

RFA is an important palliative therapy for patients with liver cancer. ${ }^{7}$ RFA uses high frequency alternating current causing focal temperatures above $60^{\circ} \mathrm{C}$ and thereby thermal necrosis. RFA 
induces thermal stress in the peritumoural area with subsequent release of pro-inflammatory cytokines such as interleukin (IL)-1, IL-6 and tumour necrosis factor $\alpha(\mathrm{TNF}-\alpha),{ }^{8}{ }^{9}$ and heat shock proteins. ${ }^{10}$ Furthermore, RFA may unmask cryptic tumour antigens that can become novel targets for antigen presenting cells (APCs) and (cytotoxic) T cells, ${ }^{11}$ as demonstrated by a robust anticancer immune response after RFA and similar ablative therapies. ${ }^{12}{ }^{13}$ This response is characterised by highly activated antitumour cytotoxic T lymphocytes (CTLs), as demonstrated in the VX2 model of liver cancer. ${ }^{14}$ However, it remained unclear how far this CTL response was primarily directed against previously immunologically cryptic tumour antigens that became unmasked upon RFA, or if the immune response would be directed against antigens from the allogeneic tumour donor. While in some studies autologous tumour tissue was used, ${ }^{15}$ others employed heterologous tumour cells. ${ }^{16}$ However, regardless of these mechanisms, stimulation of immune responses to primary or secondary hepatic malignancies by thermoablative therapies should be more effective when combined with further immunomodulatory antitumoural strategies.

The relevance of immune surveillance in the host's efficient response to various cancers has gained increasing attention and is currently exploited in ex vivo and in vivo immunostimulatory approaches, predominantly in melanoma and renal cell carcinoma, including pulsed dendritic cells (DCs) and loco-regional application of cytokines. ${ }^{17-19}$ Examples are tumour antigenspecific $T$ cell vaccination and/or vaccination by pulsed DCs in patients with melanoma, ${ }^{17} 2021$ or the systemic or loco-regional application of cytokines for immune modulation in patients with kidney cancer.

DC activation is particularly attractive since DCs can present tumour antigens to cytotoxic $\mathrm{T}$ cells, which may cause tumour destruction. ${ }^{22}$ The efficiency of tumour antigen presentation on DCs can be enhanced by stimulation of innate immune receptors, especially certain toll like receptors (TLRs) that are abundantly expressed on DCs and that can be specifically addressed by synthetic small molecular agonists. ${ }^{23}{ }^{24}$ TLR9, which recognises CpG motifs of unmethylated bacterial DNA, is an interesting but incompletely studied target to promote an antitumour cytotoxic T cell response. TLR9 is located intracellulary on endosomes and expressed in DCs or other APCs such as macrophages, monocytes, B and NK cells. ${ }^{23}$

CpG-oligodeoxynuleotides (CpG-ODN) are synthetic bacterial sequence derived nucleotides with a phosphorothioate backbone and repetitive CpG motifs, making them direct TLR9 agonists that are used clinically as adjuvants to vaccines for potentiating immunogenicity of antigens. ${ }^{25}$

The aim of this study was to investigate the effect of a combination of RFA and the TLR9 agonist CpG B on survival, tumour spread and the antitumoural cytotoxic $\mathrm{T}$ cell responses in rabbits with the highly aggressive VX2 tumour model.

\section{MATERIAL AND METHODS Oligonucleotide $\mathrm{CpG}$ and radionuclides}

ODN CpG Class B nucleotides (sequence: $5^{\prime}$-tcgtcgttgtcgttttgtcgtt3'; bold letters: $\mathrm{CpG}$ dinucleotides, regular letters: phosphorothioate linkages)) for rabbits were obtained from Coley Pharmaceuticals/Pfizer (Düsseldorf, Germany). Radionuclides were obtained from Amersham/GE Healthcare (Freiburg, Germany).

\section{ETHICAL APPROVAL}

Ethical approval for the animal experiments was obtained from the state government of Franconia (Ansbach, Government of
Franconia, Ethical Commission) and the animal use committee of the University of Erlangen-Nuremberg. All procedures were performed according the governmental guidelines of the state administration of Bavaria for experiments in animals.

\section{ANIMALS AND TUMOUR MODEL}

For this study, 70 female New Zealand white rabbits (weight $2.7 \mathrm{~kg}$; age 3-4 months; Charles River, Kimberg, Germany) were treated as outlined below, and six female rabbits were used for passaging of the VX2 tumour model. Animals were housed in the local animal facility in single cages for rabbits (Charles River, Kimberg, Germany) with food and drinking water ad libitum. A $12+12 \mathrm{~h} \mathrm{dark/light} \mathrm{cycle} \mathrm{was} \mathrm{provided} \mathrm{by} \mathrm{an} \mathrm{auto-}$ mated system. After all surgical procedures, metamizol $(5 \mathrm{~g} / \mathrm{L}$ drinking water) as analgesic was added to the drinking water for the following 5 days. The $1 \mathrm{~mm}$ tumour specimens were implanted into the middle lobe adjacent to the gall bladder under Glisson's capsule as described in prior studies of our group. ${ }^{14}{ }^{26}$ Glisson's capsule was closed by fibrin glue (Johnson, Germany) to avoid peritoneal spread.

Only tumour-bearing animals with a total tumour diameter of $9-12 \mathrm{~mm}$ at the implantation site on day 20 after implantation, as confirmed by ultrasonography, were included in the study. Animals with signs of ascites as indicator of peritoneal spread were excluded.

Randomisation into control and treatment groups was done by an independent scientist who had no relation to the present study on day 20 after implantation.

Surgical procedures and postinterventional care were performed as described before. ${ }^{14}$

\section{Cell preparation for VX2 tumour cell for intravenous injection and cell culture}

Solid VX2 tumour chips were pushed through a sterile steel strainer (mesh size $400 \mu \mathrm{m}$ ) and washed with PBS. The cell suspension was separated by gradient centrifugation using Percoll (GE Healthcare, Freiburg, Germany), followed by two washes with PBS. Cell concentration was adjusted to $10^{6}$ cells $/ \mathrm{mL}$.

\section{Study design}

A preliminary trial was performed on 18 animals to find the most effective dose of CpG B. All of these animals were transplanted intrahepatically with tumour tissue and divided into groups receiving 200, 400 or $600 \mu \mathrm{g} / \mathrm{kg}$ body weight of CpG B by subcutaneous injection three times weekly from day 21 on after tumour implantation. The animals were monitored daily and sacrificed no later than 120 days after tumour implantation. The definitive study involved 52 animals (figure 1). All animals were sacrificed 120 days after implantation of the VX2 hepatoma and treated at day 21, except for group B*. Group A (tumour implantation without any consecutive therapy, $\mathrm{n}=10$ ) and group B (treatment with RFA only 21 days after tumour implantation, $\mathrm{n}=10$ ) served as controls.

After RFA procedure, animals were randomised by an independent scientist who had no relations with the treatment of animals by choosing by lot whether receiving additional $\mathrm{CpG}$ application or staying without any additional therapy.

Group C $(\mathrm{n}=10)$ was treated with CpG B $(400 \mu \mathrm{g} / \mathrm{kg}$ body weight) and group $\mathrm{D}(\mathrm{n}=10)$ received RFA and regular subcutaneous injections of CpG B $400 \mu \mathrm{g} / \mathrm{kg}$ body weight three times weekly. Groups $\mathrm{B}^{*}$ and $\mathrm{D}^{*}(\mathrm{n}=6)$ were treated as groups $\mathrm{B}$ and $\mathrm{D}$, respectively, with RFA, but received another injection of $10^{6}$ VX2 tumour cells intravenously at day 120 to be observed for another 40 days. All animals that refused both solid and fluid 


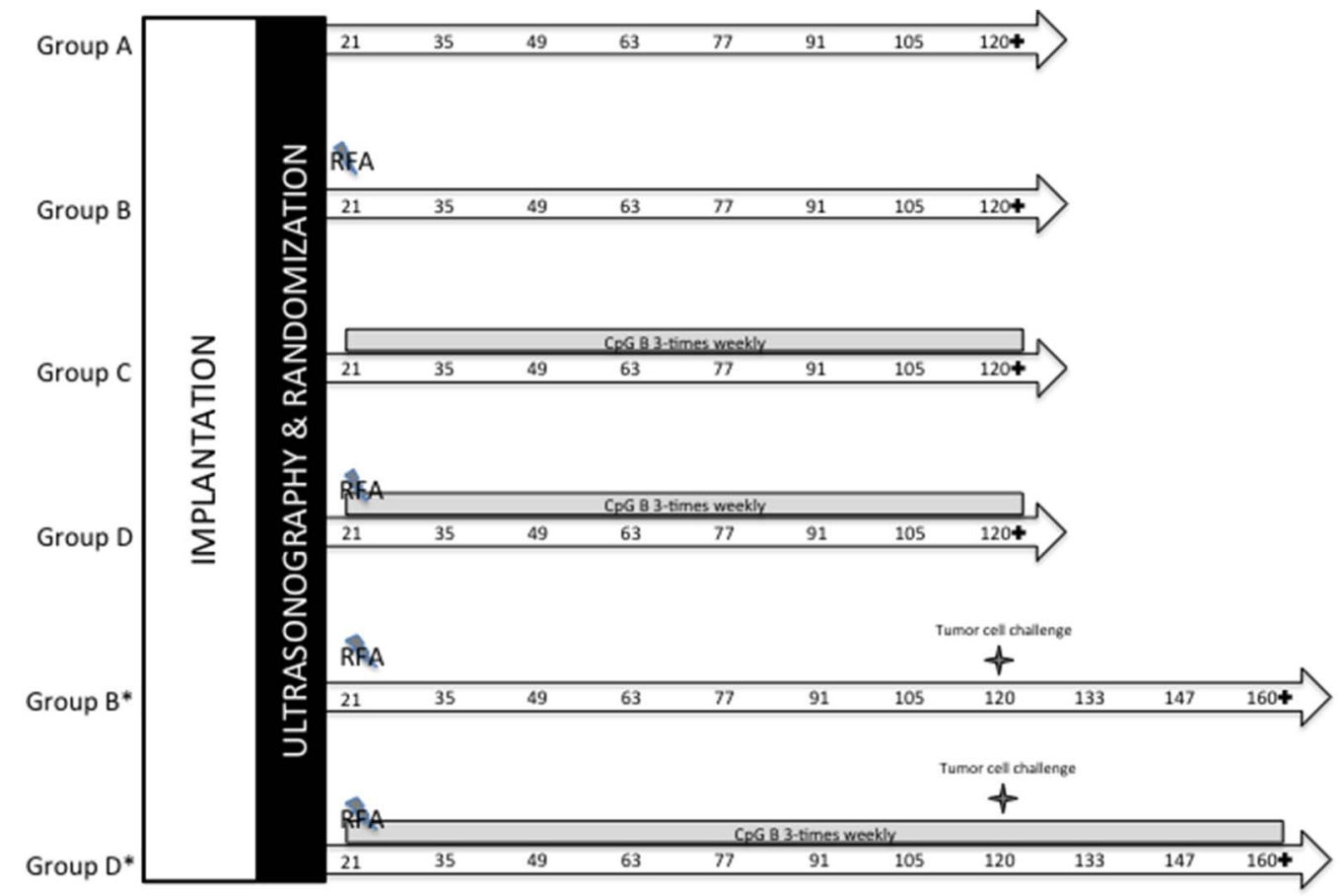

Figure 1 Study protocol. In all, 52 New Zealand white rabbits with confirmed tumour growth $(9-12 \mathrm{~mm})$ by ultrasonography on day 20 after implantation and after exclusion of peritoneal spread (like ascites) were randomised into six groups. Ten animals each were assigned to groups A-D. For subgroups $B^{*}$ and $D^{*}$, an additional six animals were included. In all animals, a subcapsular VX2 tumour was implanted into the liver. Subgroups $B^{*}$ and $D^{*}$ received a further intravenous tumour cell injection 120 days after tumour implantation. Group $A$ was left without therapy and served as control. After 21 days of tumour growth, animals of groups $B, D, B^{*}$ and $D^{*}$ were treated with radiofrequency ablation (RFA). Groups B and $B^{*}$ received no further treatment thereafter. Groups $C, D$ and $D^{*}$ were injected with $C p G$ ODN three times weekly until the end of observation. Groups A-D were autopsied 120 days and subgroups $B^{*}$ and $D^{*} 160$ days after tumour implantation. RFA treatment; numbers denote days of blood sampling after tumour implantation; +, sacrifice of animals; intravenous tumour cell injection; grey bar: period of CpG ODN treatment.

intake for more than 4 days and which showed apathy and weight loss of more than $15 \%$ were sacrificed before reaching the endpoint and were considered early deaths.

At sacrifice, animals were sacrificed to determine primary tumour, metastatic spread and histology of tumour and peritumoural regions. In all groups, samples of peripheral blood were taken from the ear vein before and 2 weeks after tumour implantation, and every 2 weeks thereafter up to day 120 (groups A-D) or day 160 (groups B* and D*) (see also figure 1).

\section{RFA APPLICATION}

After a maturation period of 21 days leading to an average tumour size of $10 \mathrm{~mm}$, animals of groups $\mathrm{B}, \mathrm{B}^{*}, \mathrm{D}$ and $\mathrm{D}^{*}$ $(n=32)$ were treated with ultrasound-guided percutaneous $\mathrm{RFA}^{14}$ using an RF generator (Elektrotom HF 106; Integra, Tuttlingen, Germany), equipped with a perfused RF needle applicator of $1.1 \mathrm{~mm}$ outer diameter and a $10 \mathrm{~mm}$ active electrode.

\section{Separation and culture of PBMC; proliferation assay and cytotoxicity of isolated PBMC}

PBMCs were isolated from citrate blood by density-gradient centrifugation in Leukosep separation tubes (PAA Laboratories, Austria) and cultured as previously described. ${ }^{14}$

A proliferation and activation assay was performed using $[3 \mathrm{H}]$ thymidine-incorporation to feeder stimulated naive PBMC as previously described. ${ }^{14}$ Phytohemagglutinine (PHA) served as positive control, fresh tumour tissue lysate as test antigen and liver tissue lysate as negative control.

To assess cytotoxicity, PBMC or serum alone were cocultured with VX2 tumour cells. Tumour cell lysis was quantified by an adenylate kinase release assay using a luciferase assay (ToxiLight Kit, Lonza, Verviers, Belgium) and expressed as relative luminescence units (RLU) according to prior studies ${ }^{27}$ (also see supplementary material and methods).

\section{Cytokine ELISA}

Serum of animals collected 14 days after tumour implantation, 4 weeks after first treatment procedure (eg, RFA, CpG application or both), was used for cytokine analysis.

ELISAs were performed according the manufacturers' guidelines and were assessed at $450 \mathrm{~nm}$ with a wave length correction at $540 \mathrm{~nm}$ to subtract background noise in a Genios plate reader (Tecan, Germany).

ELISA KITs from Antibodies-onlie, Aachen, Germany, were used for IL-2, IL-6, IL-8, IL-10, IL-12, TNF- $\alpha$, vascular endothelial growth factor (VEGF) and interferon $\gamma$ (IFN $\gamma$ ) (also see online supplementary material and methods).

\section{Histology/immunohistology}

Histological analysis was performed independently by two pathologists (DN and RK). The complete liver lobe bearing the primary tumour including adjacent healthy liver tissue, heart, lungs, liver and all metastases (lymph nodes, pleura, peritoneum) were stored in $10 \%$ buffered formalin. The $5 \mu \mathrm{m}$-thick sections were embedded in paraffin and examined after staining

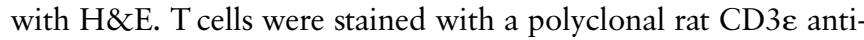
body diluted 1:10 (kind gift of Dr E Kremmer, GSF-National Research Center for Environment and Health, Munich, Germany) ${ }^{28}$ as described. ${ }^{29}$ Tris-buffered saline instead of primary antibody served as negative control. 
Stained sections were digitalised using the ImageAccess 9 Enterprise software (Imagic, Glattbrugg, Switzerland) and evaluated by counting the number of CD3 + cells per 10 high power fields.

\section{Statistical evaluation}

Statistical evaluation was done with Microsoft Excel 2008 for MAC (Microsoft Corporation, Seattle, Washington, USA) and SPSS V.15.0 (SPSS Inc, Chicago, Illinois, USA) software packages. Survival was analysed with the Kaplan-Meyer method comparing survival curves by log-rank test. The significance of all of the data was proven using the Mann-Whitney $U$ test and the ANOVA F test, respectively, and $\mathrm{p}<0.05$ was considered significant.

\section{RESULTS}

\section{Dose finding for CpG B}

Animals that received $400 \mu \mathrm{g} / \mathrm{kg} \mathrm{CpG} \mathrm{B} \mathrm{three} \mathrm{times} \mathrm{weekly} \mathrm{sur-}$ vived 75.4 days (SD 15.6) compared with 65.3 days (SD 16.9) with $200 \mu \mathrm{g} / \mathrm{kg}$ and 71.2 days (SD 12.3) with $600 \mu \mathrm{g} / \mathrm{kg}$, and 34.2 days (SD 9.4) for untreated animals. No significant differences in primary and metastatic (lung, peritoneum, lymph nodes) tumour growth were visible in any group (data not shown). Therefore, $400 \mu \mathrm{g} / \mathrm{kg}$ was chosen for further investigations.

\section{Main study: survival and tumour spread}

Data are summarised in figures 1 and $2 \mathrm{~A}, \mathrm{~B}$ and table 1 . The untreated control group A $(n=10)$ reached a mean survival of only 35.9 days (range 31-42) after tumour implantation. All animals 10/10 were considered early deaths. All animals had primary and secondary malignant lesions (lungs, peritoneum and lymph nodes). Group B $(n=10)$, which was treated with RFA 21 days after tumour implantation, reached a mean survival of 97.3 days (range 76-120), and only two animals survived
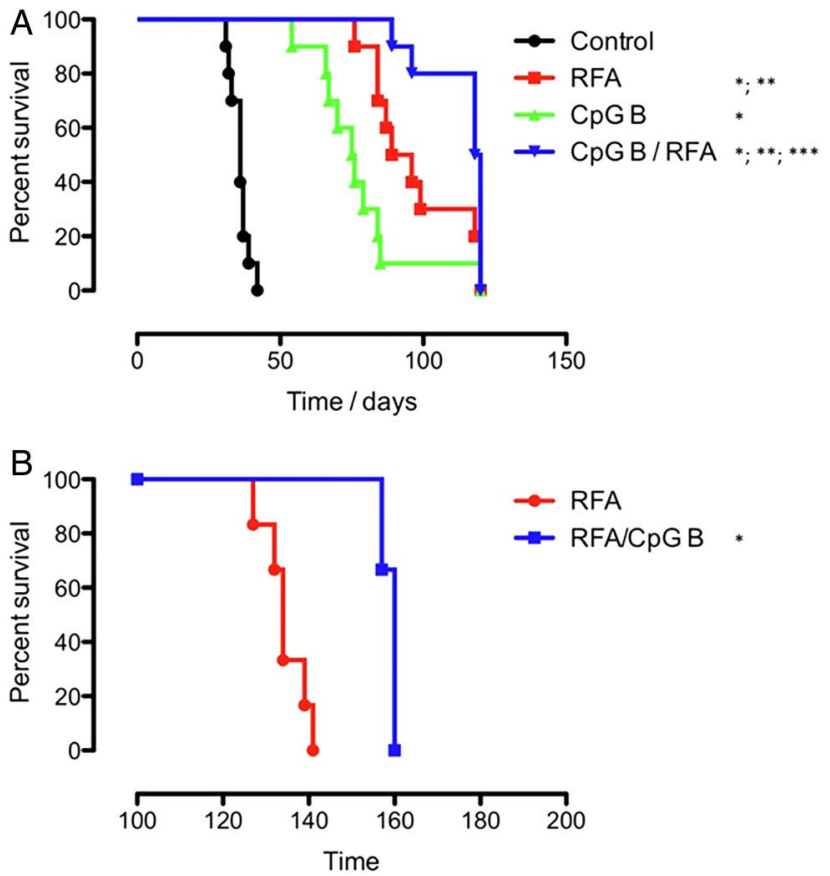

Figure 2 Kaplan-Meier survival curves for groups A-D. Log-rank ratios of survival curves of animals treated with radiofrequency ablation (RFA), CpG B, RFA/CpG B or left without treatment. * $p<0.05$ versus controls; ${ }^{* *} p<0.05$ versus CpG B only; ${ }^{* *} p<0.05$ versus RFA only.
Table 1 Overview of survival time and autoptic findings of all animals

\begin{tabular}{|c|c|c|c|c|c|}
\hline Group & No./ID & Survival/days & Primary & Pulmonary & Peritoneum \\
\hline \multirow[t]{10}{*}{ A/Control } & 1 & 42 & $x$ & $x$ & $\mathrm{x}$ \\
\hline & 2 & 37 & $x$ & $\mathrm{x} /-$ & $x$ \\
\hline & 3 & 36 & $x$ & $x$ & $x$ \\
\hline & 4 & 39 & $x$ & $x$ & $x$ \\
\hline & 5 & 32 & $x$ & $\mathrm{x} /-$ & $x$ \\
\hline & 6 & 36 & $x$ & $\mathrm{x}$ & $x$ \\
\hline & 7 & 37 & $x$ & $\mathrm{x} /-$ & $\mathrm{X} /-$ \\
\hline & 8 & 31 & $x$ & $\mathrm{x}$ & $x$ \\
\hline & 9 & 36 & $x$ & $x$ & $x$ \\
\hline & 10 & 33 & $x$ & $x$ & $x$ \\
\hline \multirow[t]{10}{*}{ B/RFA } & 1 & 84 & $x$ & $x$ & $x$ \\
\hline & 2 & 96 & - & $x$ & - \\
\hline & 3 & 120 & - & - & - \\
\hline & 4 & 118 & - & $x /-$ & $x$ \\
\hline & 5 & 99 & $\mathrm{x} /-$ & $x$ & - \\
\hline & 6 & 76 & $x$ & $x$ & $x$ \\
\hline & 7 & 120 & $\mathrm{x} /-$ & - & $x$ \\
\hline & 8 & 89 & - & $\mathrm{x} /-$ & $x$ \\
\hline & 9 & 84 & - & - & $x$ \\
\hline & 10 & 87 & - & $x$ & - \\
\hline \multirow[t]{6}{*}{ B*/RFA reexp. } & 1 & 134 & - & $x$ & - \\
\hline & 2 & 127 & - & $x$ & - \\
\hline & 3 & 139 & $x$ & $x$ & - \\
\hline & 4 & 132 & - & $x$ & - \\
\hline & 5 & 134 & $x$ & $x$ & $x$ \\
\hline & 6 & 141 & $x$ & $x$ & $x$ \\
\hline \multirow[t]{10}{*}{ C/CpG B } & 1 & 76 & $x$ & - & $x$ \\
\hline & 2 & 66 & $x$ & $x$ & $x$ \\
\hline & 3 & 54 & $x$ & $x$ & $x$ \\
\hline & 4 & 120 & - & - & - \\
\hline & 5 & 79 & $x$ & - & $x$ \\
\hline & 6 & 84 & $x$ & - & - \\
\hline & 7 & 67 & - & $\mathrm{x} /-$ & $x$ \\
\hline & 8 & 70 & $x$ & - & $x$ \\
\hline & 9 & 75 & $x$ & - & $x$ \\
\hline & 10 & 85 & $x$ & - & $x$ \\
\hline \multirow[t]{10}{*}{$\mathrm{D} / \mathrm{RFA}+\mathrm{CpG} \mathrm{B}$} & 1 & 89 & $x$ & $\mathrm{x} /-$ & - \\
\hline & 2 & 118 & - & - & - \\
\hline & 3 & 120 & - & - & - \\
\hline & 4 & 120 & - & - & - \\
\hline & 5 & 120 & - & - & - \\
\hline & 6 & 120 & - & - & - \\
\hline & 7 & 118 & - & - & - \\
\hline & 8 & 118 & - & - & - \\
\hline & 9 & 96 & $x$ & - & $x$ \\
\hline & 10 & 120 & - & - & - \\
\hline$D^{*} / \mathrm{RFA}+\mathrm{CpG}$ & 1 & 157 & - & $x$ & - \\
\hline \multirow[t]{5}{*}{ B reexp. } & 2 & 160 & - & - & - \\
\hline & 3 & 160 & - & - & - \\
\hline & 4 & 157 & $x$ & - & - \\
\hline & 5 & 160 & - & - & - \\
\hline & 6 & 160 & - & $x$ & - \\
\hline
\end{tabular}

until the end of observation and $7 / 10$ had to be sacrificed according to the protocol considering early death. Only one animal was free of malignant tissue, while nine suffered from either primary and/or secondary malignancy (to lungs and/or peritoneum and lymph nodes). Group $\mathrm{C}$ received $\mathrm{CpG} B$ three times weekly after tumour implantation. Mean survival was 77.6 days (range 54-120). In all, 1/10 animal survived until the end of observation, while $9 / 10$ animals had to be sacrificed before. Only one animal was free of malignancy, but there were 
significantly less pulmonary metastases compared with group B receiving RFA alone (CpG B 3/10 vs RFA 7/10; $\mathrm{p}<0.01)$. Animals of all groups with peritoneal spread also developed metastasis within peritoneal lymph nodes.

Group D received RFA followed by CpG B three times weekly throughout the observation period. The outcome in these animals was significantly better than in the other groups: mean survival was 113.9 days (range 89-120). Overall, 2/10 animals had to be sacrificed before reaching the end of observation. At 120 days after first treatment, 8/10 animals were free of any malignant tissue, $1 / 10$ had primary tumour and pulmonary metastases, and 1/10 primary tumour and peritoneal metastases.

After 120 days, 12 extra animals treated according to groups $B$ and D (named group $B *(n=6)$ and $D *(n=6))$ were screened for no signs of progressive malignancy (a tumour of $<1 \mathrm{~cm}$ at the implantation site as assessed by ultrasonography, lack of clinical signs of advanced tumour burden like weight loss or reduced food intake) were selected to receive an intravenous injection of VX2 tumour cells and observed for another 40 days (groups $\mathrm{B}^{*}$ and $\mathrm{D}^{*}$ ). The survival of group $\mathrm{B}^{*}$, which had an initial RFA application at day 21 after tumour implantation, was 134.5 days (range 127-141); no animal reached the end of observation. All animals developed pulmonary metastases, 2/6 had peritoneal metastases, but $3 / 6$ had no remaining tumour at the primary implantation side (figure 2A, table 1). All these tumour related parameters were further and significantly reduced in group $D^{*}$ compared with group $\mathrm{B}^{*}(\mathrm{p}<0.01$; figure 2B, table 1).

\section{Tumour-specific activation of PBMC}

Control group A, left without therapy after tumour implantation, displayed no tumour-specific activation of peripheral $\mathrm{T}$ cells (PBMC, stimulation index (SI) 2.01, SD 0.63), whereas all treated animals showed significant stimulation.

Group D, which received the combination of RFA and CpG $\mathrm{B}$, showed the highest tumour-specific lymphocyte activation $\left(\mathrm{SI}_{\mathrm{RFA} / \mathrm{CpG} \mathrm{B}}=103.43, \mathrm{SD} 55.55\right)$ compared with the other treatment groups $\left(\mathrm{SI}_{\mathrm{RFA}}=88.49, \mathrm{SD} 39.30 ; \mathrm{SI}_{\mathrm{CpG}}{ }_{\mathrm{B}}=80.75\right.$, $\mathrm{SD} 35.74$ for the groups receiving $\mathrm{CpG}$ or RFA as single therapy, respectively; $\mathrm{p}<0.05$ ) (figure $3 \mathrm{~A}$ ).

In the animals that also received intravenous injections of tumour cells (groups $\mathrm{B}^{*}$ and $\mathrm{D}^{*}$ ), T cell stimulation was even higher $\quad\left(\mathrm{SI}_{\mathrm{RFA} / \mathrm{CpG}} \quad{ }_{\mathrm{B}}=145.47, \quad \mathrm{SD} 57.35 ; \quad \mathrm{SI}_{\mathrm{RFA}}=133.37\right.$, SD 27.92; $\mathrm{p}<0.05$ ) (figure $3 \mathrm{~B}$ ).

\section{Cytotoxicity}

There was no significant lytic activity of isolated PBMC against cultured VX2 tumour cells before tumour implantation or before treatment in all groups, and in control group A throughout the whole observation period (data not shown). Two weeks after RFA and/or CpG B application, cytolytic activity of PBMCs to VX2 tumour cells significantly rose by $10-15$-fold, whereas no cytolysis was detectable in VX2 tumour cells cocultured with autologous rabbit serum.

The highest cytolytic activity of PBMC was detected in animals that received the combination of RFA combined with CpG B, while the lowest activity was found in animals which were treated with CpG B monotherapy $\left(\mathrm{RLU}_{\mathrm{RFA} / \mathrm{CpG} \mathrm{B}}=972.72\right.$, SD 362.77; $\mathrm{RLU}_{\mathrm{RFA}}=487.79$, SD 148.69; $\mathrm{RLU}_{\mathrm{CpG} \mathrm{B}}=344.52$, SD 93.81, for groups $\mathrm{B}, \mathrm{C}$ and $\mathrm{D}$, respectively; $\mathrm{p}<0.001$; figure $3 \mathrm{C}$ ). Similar results were obtained for groups $\mathrm{B}^{*}$ and $\mathrm{D}$ * that had received an intravenous injection of VX2 tumour cells (figure 3D) (no statistical difference vs animals without further tumour cell injection (groups B and D)).

\section{Histology/immunohistology}

Implanted tumours treated with RFA (group B), CpG B (group C) or their combination (group D) showed a more pronounced fibrotic margin with a mixed inflammatory infiltrate containing mononuclear cells and neutrophils compared with untreated controls (group A). Additionally, the extent of central tumour necrosis increased from CpG B or RFA treatment to their combination. There were dense infiltrates of CD3 + lymphocytes in the centre of the primary tumour in all treatment groups (B-D), regardless of the type of treatment. CpG B application led to enhancement of CD3 + lymphocytes within and at the periphery of the tumours as compared with RFA treated tumours (figure 4), and CD3 + lymphocyte infiltration was significantly more pronounced in the combination treatment group $\mathrm{D}$ than in groups $B$ or $C(p<0.05$, figure 4$)$.

\section{Cytokines}

Cytokines were measured from serum as indicated (figure 5A-H). The pro-inflammatory Th1 related cytokines IL-2, TNF- $\alpha$, IFN $\gamma$ and IL-12 were strongly upregulated in animals receiving CpGs, RFA or their combination (figure $5 \mathrm{~B}, \mathrm{D}, \mathrm{G}, \mathrm{H}$ ). Only animals treated with $\mathrm{CpG}$ alone or left untreated showed significantly elevated levels of the immunosuppressive/tolerigenic cytokine IL-10, which in combination with RFA were suppressed (figure 5F).

Pro-inflammatory IL-6 was significantly upregulated in animals receiving the combination of RFA and CpG treatment compared with animals receiving RFA treatment alone (RFA vs RFA/CpG $p<0.001$ and RFA vs control $p>0.0001$ ) while the immunostimulatory effect of $\mathrm{CpG}$ only has not met a significant level of different values compared with controls (figure 5A).

Proangiogenic VEGF and IL-8 were significantly elevated in animals receiving RFA alone and especially together with $\mathrm{CpG}$ 4 weeks after treatment (for VEGF: groups B/D; RFA vs CpG/ RFA $\mathrm{p}<0.001 ; \mathrm{CpG} / \mathrm{RFA}$ vs $\mathrm{CpG}$ alone $\mathrm{p}<0.001$; RFA vs $\mathrm{CpG}$ only $\mathrm{p}<0.0001$ ) (figure $5 \mathrm{C}, \mathrm{E}$ ).

\section{CONCLUSIONS}

Here, we tested if predominant stimulation of APCs by the TLR9 activator CpG B can potentiate RFA-mediated destruction of the VX2 tumour in rabbits, a model of aggressive and metastatic liver cancer. ${ }^{30}$ In addition, we examined how far effective primary tumour control would inhibit metastatic tumour spread to lungs, peritoneum and lymph nodes. Results of tumour control were correlated with tumour-specific $\mathrm{T}$ cell stimulation and cytotoxic $\mathrm{T}$ cell responses. Furthermore, the data explored the nature of the previously described tumour-specific immune response after RFA. ${ }^{14}$

In line with our prior data ${ }^{12} 14$ and findings from other groups, ${ }^{31}$ on the effect of RFA alone, thermoablation triggered activation of tumour-specific (cytotoxic) lymphocytes in peripheral blood and in the tumour tissue itself, as we could demonstrate with highly elevated SIs and cytolytic activities against tumour cells ex vivo, a robust $\mathrm{T}$ cell infiltrate in and around the primary tumours, and lowered tumour burden and enhanced survival in the RFA treated animals. However, these effects are still insufficient to achieve tumour control or to completely protect animals against a rechallenge of lethal tumour cells, as shown by others, ${ }^{31}{ }^{32}$ where ovalbumin (OVA) transfected melanoma cells were used, a model that is expected to be more immunogenic than a syngenic tumour cell line without specific antigen modification. Therefore, although RFA seems to generate a broader spectrum of tumour antigens for the recruitment of antigen- 

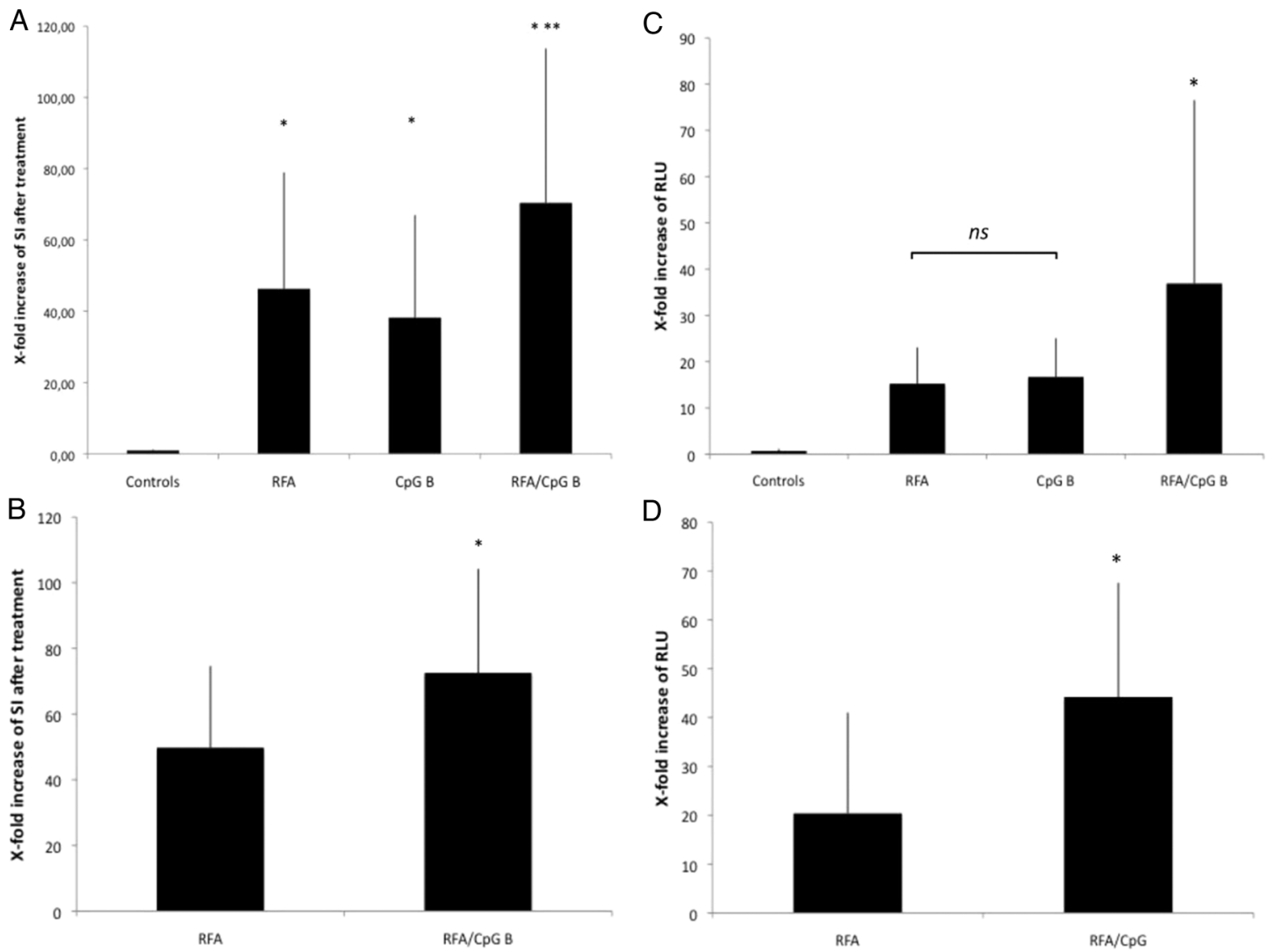

Figure 3 Tumour-specific immune response in peripheral blood. (A, B) Relative increase of stimulation indices (SIs) of PBMC exposed to tumour lysate compared with baseline stimulation (before treatment). SI was assessed by $3 \mathrm{H}$-thymidine incorporation of isolated PBMC. Baseline was assessed before treatment (21 days after tumour implantation). (A) Mean increase of SI in groups A-D. Animals were treated as indicated. (B) Mean increase of $S I$ in subgroups $B^{*}$ and $D^{*}$. All animals received an additional systemic injection of viable tumour cells intravenously 120 days after first tumour inoculation and were treated as indicated. ${ }^{*} p<0.05$ to controls; ${ }^{* *} p<0.05$ to radiofrequency ablation (RFA) or CpG monotherapy. (C, D) Relative increase of cytotoxicity of isolated PBMC co-incubated with viable tumour cells. Cytotoxicity was assessed by adenylate kinase release and expressed as relative light units (RLU). Baseline was assessed before treatment 21 days after tumour implantation. (C) Mean increase of RLU in groups A-D. Animals were treated as indicated and were autopsied 120 days after tumour implantation. (D) Mean increase of RLU in subgroups B* and $D^{*}$. All animals received an additional systemic injection of viable tumour cells intravenously 120 days after first tumour inoculation and were treated as indicated. ${ }^{*} \mathrm{p}<0.05$ to controls; NS, not significant; ${ }^{*} \mathrm{p}<<0.05$ to RFA or CpG monotherapy.

specific lymphocytes, their capacity to finally control tumour growth remains limited.

The use of vaccine adjuvants has become a widespread strategy to augment specific immune responses. Major mediators of such adjuvant effects are the TLRs, which are prominently expressed on professional APCs. ${ }^{33}$ Notably and unexpectedly, there are only few studies on the adjuvant effect of TLR9 in cancer settings. 3435

Biological response modifiers have been shown to have significantly protective effects after RFA in in vivo studies. Therefore, attenuated antigens from streptococcus pyogenes (OK-432) have been tested successfully in several animal studies. The adjuvant treatment with OK-432 was able to prolong survival nearly by two times in a VX2 tumour model in rabbits to liver or lungs, even when rechallanged by tumour cells to the ears. ${ }^{36} 37$

Recognition of bacterial DNA by TLR9 is mediated by oligomeric, unmethylated CG dinucleotides in particular sequence contexts (CpG motifs). ${ }^{38}$ Synthetic oligodeoxynucleotides (ODNs) containing such $\mathrm{CpG}$ motifs (CpG ODNs) mimic bacterial DNA and induce a coordinated set of innate immune responses, such as NFkB-mediated release of IL-8, IL-12 and consequent enhanced acquired Th1-mediated cellular and humoral immunity. ${ }^{39}$ Accordingly, in mice and in non-human primates, CpG ODNs can boost the efficacy of vaccines against bacterial, viral and parasitic pathogens, including enhancement of CTL responses in mice. ${ }^{34} 35$ $\mathrm{CpG} \mathrm{B}$, which has a phosphorothioate backbone and lacks poly-G tails, strongly promotes the maturation and activation of plasmacytoid DCs but induces only a minor IFN $\gamma$ response. ${ }^{39}$ Recent data suggest that plasmacytoid DCs and B cells are the only cells among human PBMCs that are directly responsive to CpG ODN, and that both $\mathrm{NK}$ cell activation and IFN $\gamma$ induction seem to be indirect effects of $\mathrm{CpG}$ ODNs mediated through plasmacytoid DCs by release of IFN $\alpha$ and IFN $\beta .^{40}$

Similar to the results of den Brok et $a^{32}$ who used cryoablation of OVA-transfected murine melanoma, we found a highly significant increase of activated tumour-specific lymphocytes in peripheral blood of animals receiving a combination of RFA with CpG $\mathrm{B}$ compared with animals receiving either RFA or CpG B alone. This correlated with a strong cytotoxic immune response to the tumour cells and was a good predictor of containment of tumour growth and spread, and of survival. Thus, in the combination therapy group, all animals survived up to 120 days after tumour 
Figure 4 Immunohistochemical analysis. Immunostaining for CD3 in native tumour implants treated with CpG B or the combination of CpG B and radiofrequency ablation (RFA). Nuclei were counterstained with haematoxylin. Margin and centre of tumour treated with a combination of CpG B and RFA showed a marked increase of CD3-positive T lymphocytes compared with tumour implants treated with CpG B alone (arrows indicate CD3-positive T lymphocytes, dotted lines delineate margins between normal liver and tumour tissue; $\times 400$; bar $50 \mu \mathrm{m}$ ).

Quantification of CD3-positive T lymphocytes per $10 \mathrm{hpf}$ (high power fields: magnification of $\times 400$ or per $0.06 \mathrm{~mm}^{2}$ using ImageAccess) confirmed that numbers of CD3-positive T lymphocytes increased significantly from tumour periphery to centre and between animals treated with $\mathrm{CpG} B$ alone and the combination of CpG B and RFA ( ${ }^{* *} p<0.01$, MannWhitney $U$ test).

\section{Cpg B}
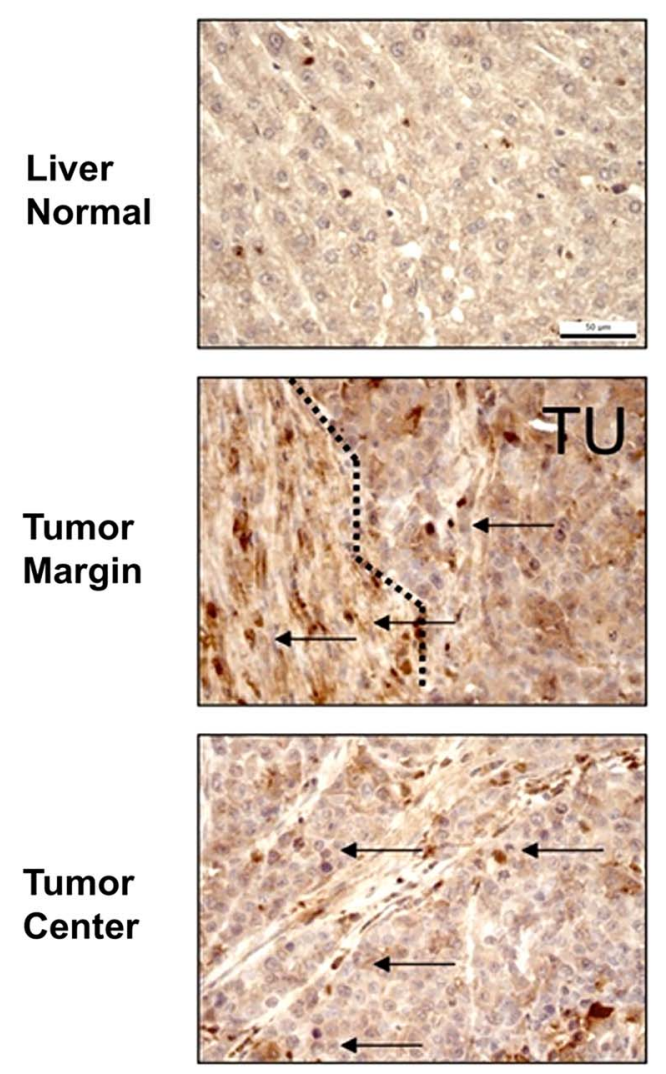

Cpg B + RFA
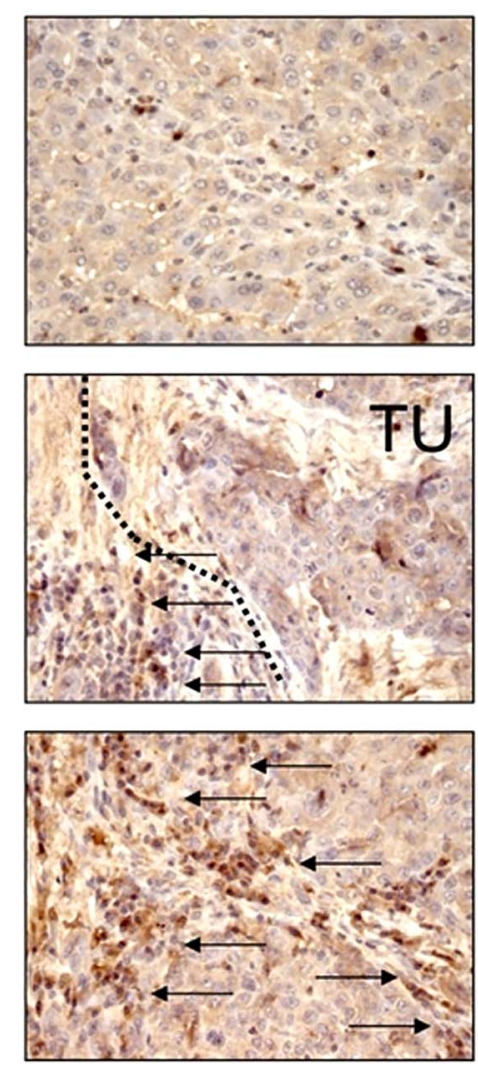

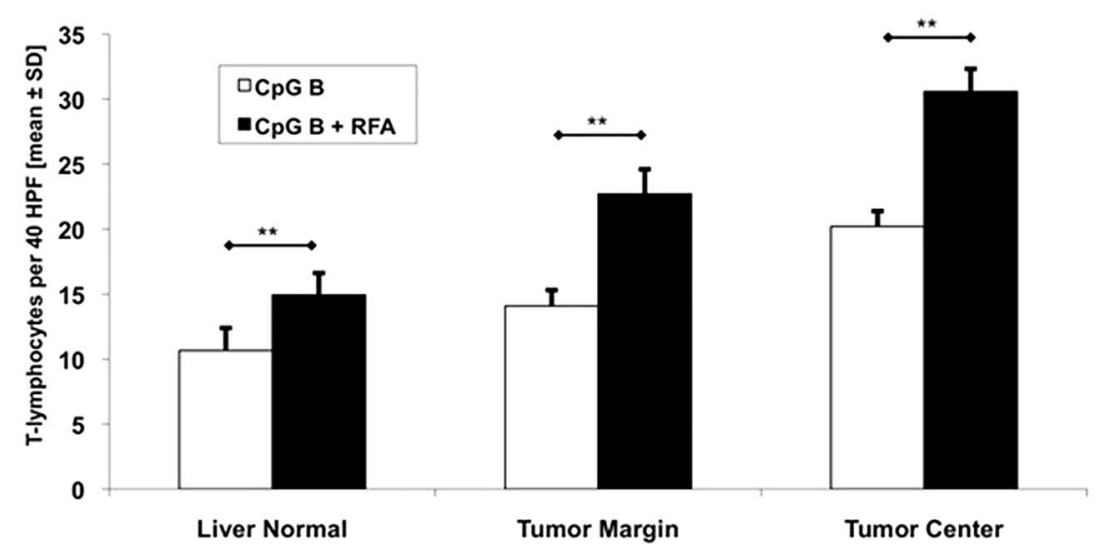

implantation as compared with a mean survival of 77.6 days (CpG B) and 97.3 days (RFA) in the monotherapy groups. Moreover, only 2/10 animals under combination therapy showed residual malignant tissue as compared with $9 / 10$ animals in each of the monotherapy groups. Upon rechallenge with viable tumour cells, only animals treated with the combination of $\mathrm{CpG}$ and RFA showed prolonged survival, in line with the previous study using the more artificial ova-transfected melanoma in a murine xenograft model. ${ }^{32}$ These authors used cryoablation combined with TLR9 stimulation, resulting in a survival rate of only 50\% after 80 days, whereas in our study combination treatment with RFA prevented secondary tumour growth in most animals (4/6) after rechallenge with malignant cells after 160 days of observation. In contrast, after tumour cell rechallenge, all animals in the RFA monotherapy group had pulmonary metastases, which are the first target for metastatic spread after an intravenous tumour seed, whereas only two of six animals in

the combination group developed pulmonary metastases and all animals of this group survived to the end of the observation period (160 days).

Various factors may be responsible for the improved outcome in our study. Apart from possible differences in the immunogenity of the tumour models, RFA triggers synthesis and presentation of clusters of co-stimulatory molecules unique to heat exposure such as heat shock proteins, ${ }^{41}$ finally leading to enhanced release of pro-inflammatory cytokines that attract APCs to infiltrate the tumour tissue and orchestrate the cellular immune response. ${ }^{42}$ This strong effect is underlined by the finding that animals treated with CpG alone showed a shorter survival than the animals treated with RFA alone. While it may be argued that the observed immune stimulation was directed against transplantation antigens rather than against prior hidden tumour antigens, our data suggest that tumour implantation alone does not elicit a detectable immune response against 

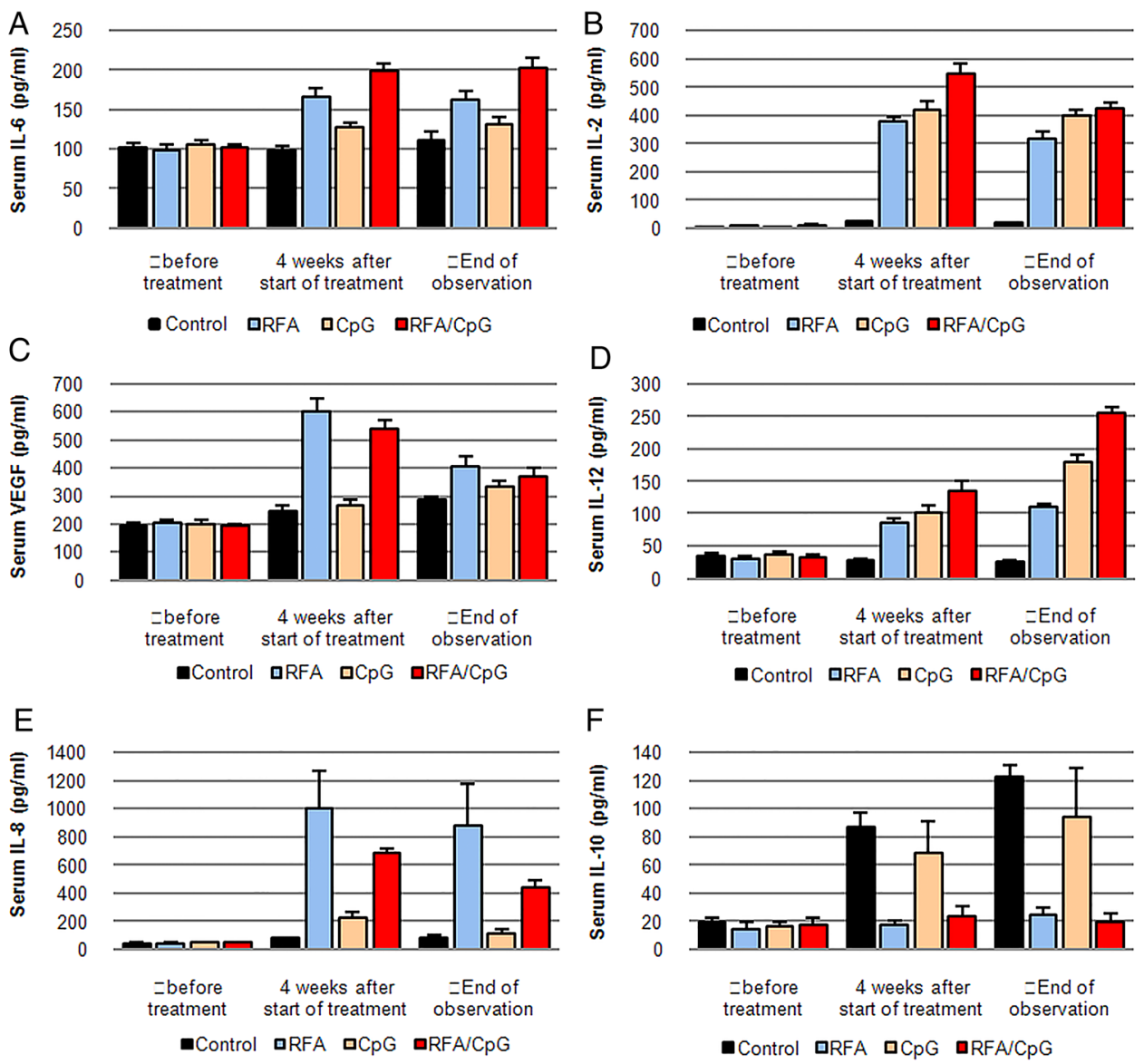

G

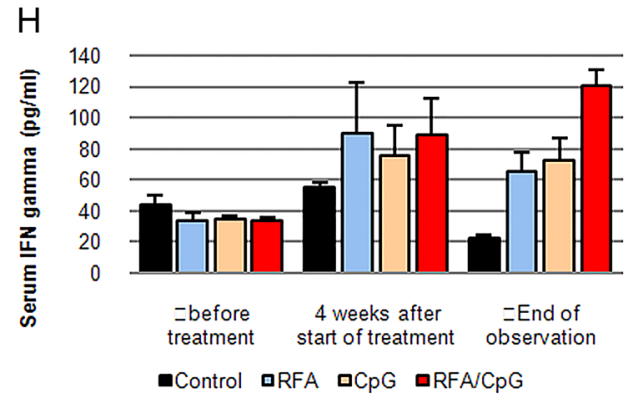

Figure 5 Cytokines in serum and tumour surrounding tissue. ELISA from blood serum. Blood was sampled as indicated. Levels of interleukin (IL)-2, IL-6, IL-8, IL-10, IL-12, interferon $\gamma$ (IFN $\gamma$ ), tumour necrosis factor $\alpha$ (TNF- $\alpha$ ) and vascular endothelial growth factor (VEGF) were measured by ELISA as detailed in Material and methods section and are expressed as relative light units (RLU). (A) IL-6: 4 weeks after treatment combination of CpG and RFA significantly highest vs. CpG or control, resp. ( $p<0.0001)$; $C p G$ vs. controls not significant $(p=0.34) ; 4$ weeks after treatment CpG/RFA significantly higher than RFA alone $(p<0.001)$; (B) IL-2: 4 weeks after treatment combination of CpG and RFA significantly highest vs. CpG or RFA alone or control, resp. ( $p<0.001$ ); At the end of observation RFA alone significantly lower vs. CpG or the combination, resp. ( $p<0.05)$. RFA/CpG vs. CpG alone without significant difference ( $p=0.42)$; (C) VEGF: 4 weeks after treatment the group receiving RFA only showed higher VEGF levels compared to all other groups which was highly significant (RFA vs. control or CpG only $p<0.0001$; RFA vs. RFA/CpG $p<0.001$ ). CpG monotherapy showed no significant differences to controls; At the end of observation VEGF levels were lowered with no significant differences between controls, RFA only or CpG monotherapy. Only the group receiving RFA alone showed significant higher levels of VEGF compared to all other groups ( $p<0.01$ ); (D) IL-12: Combination of CpG and RFA significantly highest vs. CpG or RFA alone or control, resp. ( $p<0.001)$; Combination of CpG and RFA significantly highest vs. CpG or RFA alone or control, resp. $(p<0.001)$; At the end of observation RFA alone significantly higher vs. CpG ( $p<0.01)$; (E) IL-8: RFA treatment alone or even combined with CpG co-treatment showed significantly highest levels of serum IL-8 compared to controls and CpG treated animals throughout the observation (RFA and/or RFA/CpG vs. controls or CpG alone, resp. $\mathrm{p}<0.0001$ ); IL-8 significantly suppressed by CpG co-treatment to RFA ( $p<0.001)$; (F) IL-10: Controls and CpG treated animals significantly higher levels vs. RFA or RFA/CpG up to the end of observation $(p<0.0001)$; $(G) T N F \alpha$ : 4 weeks after treatment all groups showed significant risen levels of serum-TNF $\alpha$ while (co)treatment with CpG ODN showed significantly higher levels compared to RFA alone (CpG or RFA/CpG vs. RFA alone $p<0.01$ ); At the end of observation there was no significant difference between RFA alone vs. controls while CpG (co)treated animals still had significantly elevated levels (CpG or CpG/RFA vs. RFA alone $\mathrm{p}<0.001) ;(\mathrm{H})$ IFN $\gamma$ : 4 weeks after treatment until the end of observation all treated groups (RFA, CpG, RFA/CpG) showed significantly risen IFN $\gamma$ levels compared to controls $(p<0.001)$ while there was no difference between the treated groups. Only at the end of observation animals receiving a combination of RFA/CpG had significantly elevated IFN $\gamma$ levels compared to animals of the mono-therapy groups (RFA/CpG vs. RFA or $C p G$, resp. $p<0.001)$. Significance was assessed by Mann-Whitney-U-Test. 
tumour cells. Equally, PBMC obtained from control animals never responded to tumour lysate.

It has been demonstrated that RFA causes local and systemic Tcell stimulation by release of stress molecules like heat shock proteins $^{43}$ and high mobility group B (HMGB-1). ${ }^{26} 4445$ However, as discussed above, this response does not seem to be strong enough to induce complete tumour rejection and prevent future metastatic spread. ${ }^{14}$ Here, CpG B can serve as an adjuvant to enhance preformed adaptive immunity against specific antigens. ${ }^{24}$

Due to the lack of antibody reagents for the VX2-tumour model in rabbits which is the major model for local ablative treatments, it was not possible to elucidate indepth histopathological changes. Nevertheless, with the available technologies, we could demonstrate significant upregulation of Th1 cytokines such as IL-2, IL-12 and IFN $\gamma$ in those settings where maximal tumour-specific $\mathrm{T}$ cell activation or cytotoxic activity was found whereas, for example, the immunosuppressive IL-10 was higher in conditions of enhanced tumour spread. Notably, CpG B alone induces higher levels in cytokines related both to a Th1 and Th2/M2, 25 indicating that it is highly important in what setting CPG B is applied. Disappointingly, our data even implicate that $\mathrm{CpG}$ treatment without a preformed directed immune response to a target even raises immunotolerance against malignant tissue in a tolorigenic M2 directed environment.

Finally, the higher VEGF levels in animals receiving RFA only are in accordance to prior findings, while an incomplete destruction of viable tumour tissue can lead to enhanced tumour growth. ${ }^{46}$ VEGF expression has previously been correlated to hypoxia, tumour associated macrophage infiltration and enhanced angiogenesis. Taken together, RFA appears to create an in vivo environment resembling $T$ cell vaccination against a broad spectrum of relevant antigens, with CpG B serving as an adjuvant that leads to further activation of pre-existing APCs and suppression of the angiogenic response.

The original discovery of CpG oligonucleotides in $1995 \mathrm{had}$ initially created enormous enthusiasm. However, several clinical trials yielded disappointing results. ${ }^{47} \mathrm{~A}$ possible explanation for this inefficiency could be the lack of a distinct (tumour derived) target. It is still a pitfall of undirected immunostimulatory cancer therapies such as $\mathrm{CpG}$ ODN treatment that these may activate a Th1 response and an immunosuppressive immune response, for example, via secretion of IL-10 from tumour associated macrophages or DCs in chronic tumour associated inflammation. 48

In conclusion, our data suggest that RFA acts as the driver of an immune response by inducing the release of ER-linked glycoproteins (such as gp96) and HSPs leading to a Th1/M1-specific immune response towards tumour antigens from the ablated tumour tissue, as indicated by the enhanced expression of IL-2 and IFN $\gamma$. CpG ODN acts as an innate adjuvant driving both Th1 and Th2T cell responses (indicated by IL-10 cytokine release). Our data implicate that the combination of a Th1 directed immune response resulting from local ablative therapies together with a systemic enhancer of this immune response could improve the outcome even in case of systemic spread of malignancy.

Since CpG ODNs are already in use as well tolerated and potent adjuvants to vaccines, the additive therapy with $\mathrm{CpG}$ ODN in combination with interventional locally ablative therapies could be promising avenue for anti-HCC therapies.

Our data implicate the cellular cytotoxic immune response as a major determinant of antitumour efficacy after RFA. In light of CpG oligonucleotides that are currently being tested in phase II and III clinical trials for non-small cell lung cancer ${ }^{49} 50$ and melan$\mathrm{oma}^{51}$ in advanced stages failing first-line therapy, it may be suggested that their unique combination with ablative therapies like RFA offers a particularly attractive treatment option for primary and secondary tumours by offering a distinct tumour destructive immune environment of thermally altered antigens and local.

\section{Author affiliations}

${ }^{1}$ Department of Medicine 1, University Hospital Erlangen-Nuremberg, Erlangen, Germany

${ }^{2}$ Institute for Surgical Research, Philipps-University Marburg, Marburg, Germany ${ }^{3}$ Division of Gastroenterology, University Hospital, Philipps-University Marburg, Marburg, Germany

${ }^{4}$ Institute of Pathology, Paracelsus Medical University, Salzburg, Austria

${ }^{5}$ Division of Gastroenterology, Beth Israel Deaconess Medical Center, Harvard Medical School, Boston, Massachusetts, USA

${ }^{6}$ Institute of Translational Immunology, University Medical Center, Mainz, Germany

Acknowledgements The present work was performed in fulfillment of the requirements in obtaining the medical degree for BB.

Contributors All authors had substantially worked in the study underlying the manuscript and/or had substantially impacted and/or worked on the design of the study: BB, PDF: experiments, study design and manuscript writing; PM, EGH, DeiS TG: study design, manuscript writing and editing; DN, RK: immunohistochemistry, statistical evaluation, manuscript writing and editing; DetS, TTW: study design, manuscript writing and editing, experiments, surgical procedures, main intellectual impact and fund raising.

Funding This study was supported by grants from the interdisciplinary centre for clinical research (IZKF), University of Erlangen, the Hans Löwel Foundation Bamberg, Germany, and NIH U19 Al066313 to DS.

Competing interests None.

Provenance and peer review Not commissioned; externally peer reviewed.

\section{REFERENCES}

1 Hanish SI, Knechtle SJ. Liver transplantation for the treatment of hepatocellular carcinoma. Oncology (Williston Park) 2011;25:752-7.

2 Schuppan D, Afdhal NH. Liver cirrhosis. Lancet 2008;371:838-51.

3 Llovet JM, Burroughs A, Bruix J. Hepatocellular carcinoma. Lancet 2003;362:1907-17.

4 Varela M, Sala M, Llovet JM, et al. Treatment of hepatocellular carcinoma: is there an optimal strategy? Cancer Treat Rev 2003;29:99-104.

5 Bruix J, Llovet JM. HCC surveillance: who is the target population? Hepatology 2003; 37:507-9

6 Llovet JM, Ricci S, Mazzaferro V, et al. Sorafenib in advanced hepatocellular carcinoma. N Engl J Med 2008;359:378-90.

7 Georgiades CS, Hong K, Geschwind JF. Radiofrequency ablation and chemoembolization for hepatocellular carcinoma. Cancer J 2008;14:117-22.

8 Gallucci S, Lolkema M, Matzinger P. Natural adjuvants: endogenous activators of dendritic cells. Nat Med 1999;5:1249-55.

9 Fuchs EJ, Matzinger P. Is cancer dangerous to the immune system? Semin Immunol 1996:8:271-80

10 Yang WL, Nair DG, Makizumi R, et al. Heat shock protein 70 is induced in mouse human colon tumor xenografts after sublethal radiofrequency ablation. Ann Surg Oncol 2004;11:399-406

11 Nobuoka D, Motomura Y, Shirakawa $\mathrm{H}$, et al. Radiofrequency ablation for hepatocellular carcinoma induces glypican-3 peptide-specific cytotoxic $T$ lymphocytes. Int J Oncol 2012;40:63-70.

12 Hansler J, Wissniowski TT, Schuppan D, et al. Activation and dramatically increased cytolytic activity of tumor specific $T$ lymphocytes after radio-frequency ablation in patients with hepatocellular carcinoma and colorectal liver metastases. World J Gastroenterol 2006;12:3716-21.

13 Napoletano C, Taurino F, Biffoni M, et al. RFA strongly modulates the immune system and anti-tumor immune responses in metastatic liver patients. Int $\mathrm{J}$ Oncol 2008;32:481-90.

14 Wissniowski TT, Hansler J, Neureiter D, et al. Activation of tumor-specific $\mathrm{T}$ lymphocytes by radio-frequency ablation of the VX2 hepatoma in rabbits. Cancer Res 2003;63:6496-500.

15 Zerbini A, Pilli M, Fagnoni $F$, et al. Increased immunostimulatory activity conferred to antigen-presenting cells by exposure to antigen extract from hepatocellular carcinoma after radiofrequency thermal ablation. J Immunother 2008;31:271-82.

16 Liu Q, Zhai B, Yang W, et al. Abrogation of local cancer recurrence after radiofrequency ablation by dendritic cell-based hyperthermic tumor vaccine. $\mathrm{Mol}$ Ther 2009;17:2049-57.

17 Alexandrescu DT, Ichim TE, Riordan NH, et al. Immunotherapy for melanoma: current status and perspectives. J Immunother 2010;33:570-90. 
18 Moschella F, Proietti E, Capone I, et al. Combination strategies for enhancing the efficacy of immunotherapy in cancer patients. Ann N Y Acad Sci 2010;1194:169-78.

19 Andersen $\mathrm{MH}$, Junker $\mathrm{N}$, Ellebaek $\mathrm{E}$, et al. Therapeutic cancer vaccines in combination with conventional therapy. J Biomed Biotechnol 2010;2010:237623.

20 Nakai N, Hartmann G, Kishimoto S, et al. Dendritic cell vaccination in human melanoma: relationships between clinical effects and vaccine parameters. Pigment Cell Melanoma Res 2010;23:607-19.

21 Nakamoto $\mathrm{Y}$, Mizukoshi E, Kitahara M, et al. Prolonged recurrence-free survival following OK432-stimulated dendritic cell transfer into hepatocellular carcinoma during transarterial embolization. Clin Exp Immunol 2011;163:165-77.

22 Kalinski P, Urban J, Narang R, et al. Dendritic cell-based therapeutic cancer vaccines: what we have and what we need. Future Oncol 2009;5:379-0.

23 Murad YM, Clay TM, Lyerly HK, et al. CPG-7909 (PF-3512676, ProMune): toll-like receptor-9 agonist in cancer therapy. Expert Opin Biol Ther 2007;7:1257-66.

24 Rothenfusser S, Hornung V, Ayyoub M, et al. CpG-A and CpG-B oligonucleotides differentially enhance human peptide-specific primary and memory CD8+ T-cell responses in vitro. Blood 2004;103:2162-9.

25 Bode $C$, Zhao G, Steinhagen $F$, et al. CpG DNA as a vaccine adjuvant. Expert Rev Vaccines 2011;10:499-511.

26 Hansler J, Neureiter D, Wasserburger $M$, et al. Percutaneous US-guided radiofrequency ablation with perfused needle applicators: improved survival with the VX2 tumor model in rabbits. Radiology 2004;230:169-74.

27 Vogl TJ, Wissniowski TT, Naguib NN, et al. Activation of tumor-specific T lymphocytes after laser-induced thermotherapy in patients with colorectal liver metastases. Cancer Immunol Immunother 2009;58:1557-63.

28 Beineke A, Siebert U, Wunschmann A, et al. Immunohistochemical investigation of the cross-reactivity of selected cell markers from various species for characterization of lymphatic tissues in the harbour porpoise (Phocoena phocoena). J Comp Pathol 2001;125:311-17.

29 Kemmerling R, Stintzing S, Muhlmann J, et al. Primary testicular lymphoma: a strictly homogeneous hematological disease? Oncol Rep 2010;23:1261-7.

30 Sonoda A, Nitta N, Nitta-Seko A, et al. Time-course studies of implanted rabbit VX2 liver tumors to identify the appropriate time for starting hepatic arterial embolization in animal models. Oncology 2011;80:92-6.

31 Dromi SA, Walsh MP, Herby $S$, et al. Radiofrequency ablation induces antigen-presenting cell infiltration and amplification of weak tumor-induced immunity. Radiology 2009;251:58-66.

32 den Brok MH, Sutmuller RP, Nierkens $S$, et al. Efficient loading of dendritic cells following cryo and radiofrequency ablation in combination with immune modulation induces anti-tumour immunity. Br J Cancer 2006;95:896-905.

33 Akira S, Takeda K, Kaisho T. Toll-like receptors: critical proteins linking innate and acquired immunity. Nat Immunol 2001;2:675-80.

34 Davila E, Celis E. Repeated administration of cytosine-phosphorothiolated guanine-containing oligonucleotides together with peptide/protein immunization results in enhanced CTL responses with anti-tumor activity. J Immunol 2000;165:539-47.
35 Heckelsmiller K, Rall K, Beck S, et al. Peritumoral CpG DNA elicits a coordinated response of CD8T cells and innate effectors to cure established tumors in a murine colon carcinoma model. J Immunol 2002;169:3892-9.

36 Kageyama K, Yamamoto A, Okuma T, et al. Radiofrequency ablation of liver tumors in combination with local OK-432 injection prolongs survival and suppresses distant tumor growth in the rabbit model with intra- and extrahepatic VX2 tumors. Cardiovasc Intervent Radiol 2013;36:1383-92.

37 Hamamoto S, Okuma T, Yamamoto A, et al. Radiofrequency ablation and immunostimulant OK-432: combination therapy enhances systemic antitumor immunity for treatment of VX2 lung tumors in rabbits. Radiology 2013;267:405-13.

38 Dalpke A, Zimmermann S, Heeg K. Immunopharmacology of CpG DNA. Biol Chem 2002;383:1491-500.

39 Krieg AM. CpG motifs in bacterial DNA and their immune effects. Annu Rev Immunol 2002;20:709-60.

40 Hornung V, Rothenfusser S, Britsch S, et al. Quantitative expression of toll-like receptor 1-10 mRNA in cellular subsets of human peripheral blood mononuclear cells and sensitivity to CpG oligodeoxynucleotides. J Immunol 2002;168:4531-7.

41 Jolesch A, Elmer K, Bendz $\mathrm{H}$, et al. Hsp70, a messenger from hyperthermia for the immune system. Eur J Cell Biol 2012;91:48--52.

42 Beere HM. "The stress of dying": the role of heat shock proteins in the regulation of apoptosis. J Cell Sci 2004;117:2641-51.

43 Nikfarjam M, Muralidharan V, Su K, et al. Patterns of heat shock protein (HSP70) expression and Kupffer cell activity following thermal ablation of liver and colorectal liver metastases. Int J Hyperthermia 2005;21:319-32.

44 Binder RJ. CD40-independent engagement of mammalian hsp70 by antigen-presenting cells. J Immunol 2009;182:6844-50.

45 Binder RJ, Kelly JB III, Vatner RE, et al. Specific immunogenicity of heat shock protein gp96 derives from chaperoned antigenic peptides and not from contaminating proteins. J Immunol 2007;179:7254-61.

46 Isbert C, Ritz JP, Roggan A, et al. Enhancement of the immune response to residual intrahepatic tumor tissue by laser-induced thermotherapy (LITT) compared to hepatic resection. Lasers Surg Med 2004;35:284-92.

47 Badie B, Berlin JM. The future of CpG immunotherapy in cancer. Immunotherapy 2013:5:1-3.

48 Dearman RJ, Cumberbatch M, Maxwell G, et al. Toll-like receptor ligand activation of murine bone marrow-derived dendritic cells. Immunology 2009;126:475-84.

49 Yamada K, Nakao M, Fukuyama C, et al. Phase I study of TLR9 agonist PF-3512676 in combination with carboplatin and paclitaxel in patients with advanced non-small-cell lung cancer. Cancer Sci 2010;101:188-95.

50 Manegold C, Gravenor D, Woytowitz D, et al. Randomized phase II trial of a toll-like receptor 9 agonist oligodeoxynucleotide, PF-3512676, in combination with first-line taxane plus platinum chemotherapy for advanced-stage non-small-cell lung cancer. J Clin Oncol 2008;26:3979-86.

51 Fourcade J, Kudela P, Andrade Filho PA, et al. Immunization with analog peptide in combination with CPG and montanide expands tumor antigen-specific CD8+ T cells in melanoma patients. J Immunother 2008;31:781-91. 